\title{
Developmental Responses of Two Substrains of in vitro Fertilized C57BL/6J Mouse Embryos to Oxygen and Amino Acids
}

\author{
Seiji KITO1), Yoshiko NOGUCHI ${ }^{2)}$ and Yuki OHTA ${ }^{2)}$ \\ ${ }^{1)}$ National Institute of Radiological Sciences, 4-6-1 Anagawa, Chiba 263-8555, and \\ ${ }^{2}$ Science Services 4-6-1 Anagawa, Chiba 263-8555, Japan
}

\begin{abstract}
The developmental response of in vitro fertilized embryos to oxygen and amino acids were compared between in-house bred C57BL/6JNrs (Nrs) and commercially available C57BL/6JSIC (SIC) mice. Under 20\% oxygen, the percentage of embryos that developed to blastocysts and expanded blastocysts, and nuclear numbers were lower in SIC embryos than in Nrs embryos. Moreover, the nuclear number did not increase in Slc embryos during 72-96 h culture. Effects of amino acids were beneficial on development of Slc embryos under $20 \%$ oxygen, but inhibitory on blastocoel formation at $78 \mathrm{~h}$ under $5 \%$ oxygen. On the other hand effects of amino acids on Nrs embryos were observed in nuclear number at 72 and $78 \mathrm{~h}$ culture under $5 \%$ oxygen. Because the present study showed differences in sensitivity to culture conditions between the C57BL/6J substrains, care must be taken in embryo manipulation of this inbred strain in studies of embryo development or other studies.
\end{abstract}

Key words: C57BL/6J inbred mice, in vitro development, mouse embryos

Embryo manipulation is a powerful and essential technique in laboratory animal science and has applications for large-scale animal production, cryopreservation and distribution of various mouse resources $[16,17]$. In vitro fertilization and embryo culture are fundamental to accomplishing proper embryo manipulation. Although it is true that under conventional culture conditions the developmental ability of mouse embryos varies among inbred strains of mice $[15,19,22]$, the developmental competence of substrains has not been studied. In the present study, we compared the embryo development of two substrains of C57BL/6J mice, namely, in-house bred C57BL/6JNrs (Nrs) and commercially available C57BL/6JSlc (Slc) mice from SLC Inc. We selected C57BL/6J mice because viable zygotes and embryos of this strain have become important materials for biological research, particularly for the production of transgenic mice by pronuclear injection and gene-deleted mice by introduction of transformed embryonic stem cells [8, 20, 24].

Inbred C57BL/6JNrs mice had been maintained by sib-mating over 80 generations in the animal facility of the National Institute of Radiological Sciences, and C57BL/6JSlc mice were purchased from SLC Inc. 
(Hamamatsu, Shizuoka, JAPAN). Animals were maintained on a $12 \mathrm{~h}$ light:12 h dark cycle with free access to food (MB-1, Funahashi Farm Co., Ltd., Chiba, Japan) and water, and were treated according to the Recommendations for Handling of Laboratory Animals for Biomedical Research, compiled by the Committee on the Safety and Ethical Handling Regulations for Laboratory Animal Experiments, the National Institute of Radiological Sciences, Japan. Females of age 8 to 12 weeks old were injected intraperitoneally with 5 IU of equine chorionic gonadotropin (eCG) followed by an injection of 5.0 IU human chorionic gonadotropin (hCG) 46-48 h later. The cumulus-oocyte complexes (COCs) were obtained from the oviducts $16 \mathrm{~h}$ after hCG injection. Spermatozoa were collected from the distal cauda epididymides and capacitated in modified Krebs-Ringer bicarbonate solution (TYH) [21] for 1.5$2 \mathrm{~h}$ at a concentration of $1.0-5.0 \times 10^{6} \mathrm{sperms} / \mathrm{ml}$ under $5 \% \mathrm{CO}_{2}$ in air at $37^{\circ} \mathrm{C}$. The drops containing the COCs were inseminated with capacitated sperm at a concentration of $1.5 \times 10^{5} \mathrm{sperms} / \mathrm{ml}$. Five hours post-insemination (PI), eggs with the second polar body and two pronuclei were collected for further culture.

The basic culture medium used in this experiment was KSOM [12]. A $2 \times 2$ factorial experimental design was applied; $5 \%$ and $20 \%$ (atmospheric oxygen) oxygen tension against the presence or absence of half strength MEM amino acids $(10 \mu \mathrm{l} / \mathrm{ml}$ essential amino acids: GIBCO BRL \#11130-051, Rockville, MD, USA, and $5 \mu \mathrm{l} / \mathrm{ml}$ non-essential amino acids: GIBCO BRL \#11140-050) [3, 7]. An equal number of eggs from each individual animal was distributed to one of the four treatment groups examined (controlled pooling) [14]. Development of embryos to the blastocyst stage and expanded blastocyst stage was scored at 72,78 and 96 h PI under a dissecting microscope. For the determination of nuclear numbers, one-third of the embryos were fixed in $1 \%$ formalin at each time point, stained with Hoechst 33342 and the nuclei were counted under fluorescence microscopy [22].

Data were collected from four replicates of the experiment. In vitro development data were transformed by the Tukey-Freeman arcsin transformation [25], and nuclear numbers by log transformation. The transformed data were subjected to analysis of variance using the SAS program and the least significant difference test was used for multiple comparisons. a. Blastocysts at $78 \mathrm{hr} \mathrm{P}$

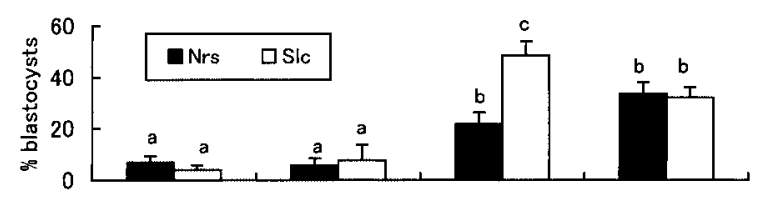

b. Blastocysts at $96 \mathrm{hr} \mathrm{P}$

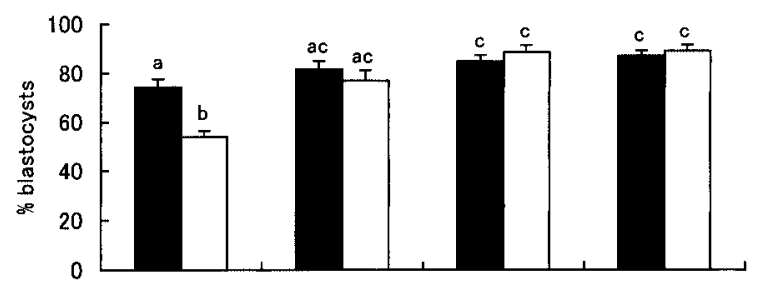

c. Expanded blastocysts at $96 \mathrm{hr} \mathrm{PI}$

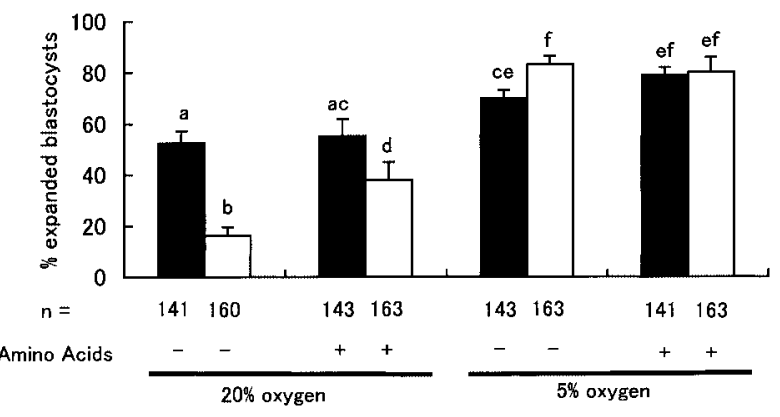

Fig. 1. In vitro development of C57/BL6J embryos of the Nrs and Slc substrains under various conditions. Results are shown as means \pm S.E.M. of four replicates of experiment. abcdef: Different letters in each figure indicate significant difference $(\mathrm{P}<0.05)$.

Effects of amino acids and oxygen on in vitro fertilized embryos were observed from 78 h PI. Few $(<2 \%)$ embryos developed to the blastocyst stage by $72 \mathrm{~h}$ PI (data not shown). Figure 1 shows the development of embryos to the blastocyst and expanded blastocyst stage at 78 (Fig. 1.a) and $96 \mathrm{~h} \mathrm{PI} \mathrm{(Fig.} \mathrm{1.b).} \mathrm{The} \mathrm{percentage}$ of embryos developing to the blastocyst stage was higher under $5 \%$ oxygen in both substrains of embryos. Under $20 \%$ oxygen, a significantly higher percentage of Nrs embryos developed to expanded blastocysts than Slc embryos (Fig. 1.c). Beneficial effects of amino acids were observed in Slc, but not in Nrs embryos at $96 \mathrm{hr}$ PI under $20 \%$ oxygen. Under $5 \%$ oxygen, a significantly higher percentage of Slc embryos (48\%) 
a. $72 \mathrm{hr} \mathrm{PI}$

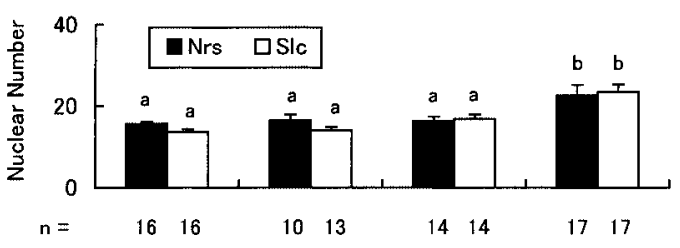

b. $78 \mathrm{hr} \mathrm{Pl}$

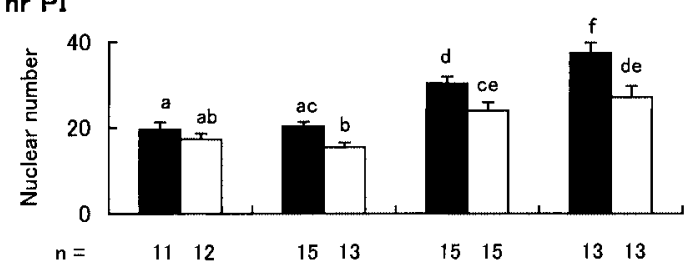

c. $96 \mathrm{hr} \mathrm{PI}$

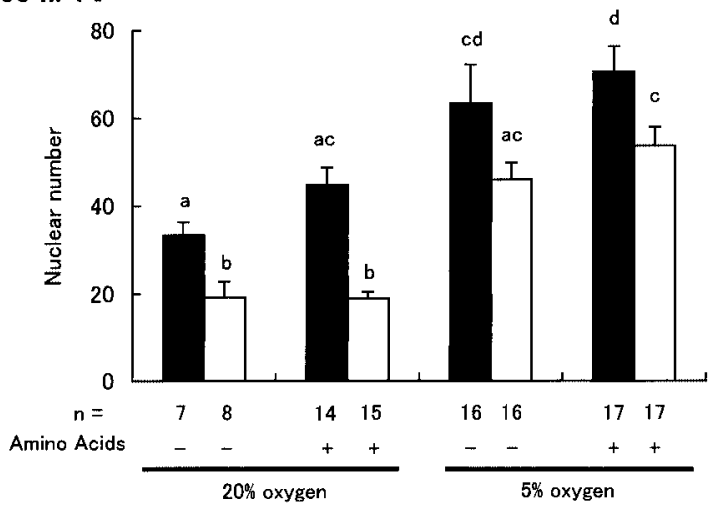

Fig. 2. Nuclear number of in vitro fertilized C57/BL6J embryos of Nrs and Slc substrains cultured under various conditions. Results are shown as means \pm S.E.M. abcd: Different letters at each time window indicate significant difference $(\mathrm{P}<0.05)$.

cultured without amino acids developed to the blastocyst stage than Nrs embryos (22\%) at 78 h PI (Fig. 1.a).

Figure 2 shows the nuclear number of embryos at 72 (Fig. 2.a), 78 (Fig. 2.b) and 96 h PI (Fig. 2.c). The nuclear number of embryos cultured under $5 \%$ oxygen tended to be higher in both substrains of embryos than that under $20 \%$ oxygen. At 96 h PI (Fig. 2.c), the nuclear number was higher in Nrs embryos than in Slc embryos under any condition. Under $20 \%$ oxygen, amino acids did not have any significant effect on the nuclear number in either substrain. Under 5\% oxygen, the nuclear number of Nrs embryos was significantly increased by supplementation of amino acids at 72 (Fig. 2.a) and $78 \mathrm{~h}$ PI (Fig. 2.b). The nuclear number of Nrs embryos increased significantly from $72 \mathrm{~h}$ to $96 \mathrm{~h}$ PI under all conditions, while no such increase was observed during the same period in Slc embryos cultured in $20 \%$ oxygen (statistical results are not shown).

Mammalian embryos are highly sensitive to oxygen tension and amino acids during in vitro culture [1, 3-5, $7,18]$. Our data confirmed previous findings $[1,3-5$, $7,18]$ on the effects of oxygen and amino acids on in vitro embryo development. In vitro developmental competence of mouse embryos has been reported to vary depending on the genetic background of particular mouse strains $[1,2,15,19,23]$. In this report, we showed that this sensitivity also varies between substrains.

The detrimental effect of $20 \%$ oxygen was prominent in Slc embryos: poor development to expanded blastocysts and no increase in the nuclear number of embryos cultured under $20 \%$ oxygen from 72 to $96 \mathrm{~h}$ PI. Although development in $20 \%$ oxygen was lower in Nrs embryos as well, more than $50 \%$ of embryos developed to the expanded blastocyst stage and there was increase in nuclear number during 78 to $96 \mathrm{~h}$ culture under all culture conditions (statistical results are not shown). These results indicate higher sensitivity of Slc embryos than Nrs embryos to oxygen tension.

Response to amino acid supplementation was complicated (Fig. 1). Under 5\% oxygen, amino acid supplementation slowed blastocoel formation of Slc embryos at $78 \mathrm{~h} \mathrm{PI}$, although amino acids had positive effects on nuclear numbers at $72 \mathrm{~h}$ PI (Fig. 2). Under $20 \%$ oxygen, the percentage of Slc embryos that developed to blastocysts and expanded blastocysts was higher in the presence of amino acids than in their absence. Lane and Gardner [11] showed that supplementation of nonessential amino acids had negative effects until the third cleavage, but was stimulatory afterwards. There is a possibility that the amino acids used in the present study are suboptimal for embryo development since we added both nonessential and essential amino acids during the entire period of embryo culture.

The difference of developmental response to culture environments described in this experiment may reflect genetic diversity among substrains. Because oxygen radicals have been shown to be one of the factors related to developmental retardation of in vitro embryo culture $[1,9]$, different responses against oxygen con- 
centration may be derived from differences in ability at removing the detrimental effects of free oxygen radicals between the two substrains. Amino acids can function as controllers of intracellular $\mathrm{pH}$ and components of glutathione [1]. We expected beneficial effects of amino acids on embryo development and nuclear numbers in combination irrespective of oxygen concentrations. However, the effects of amino acids and their interaction with oxygen concentrations showed conflicting results in this study as in Slc embryos amino acids inhibited blastocoel formation but were beneficial for nuclear numbers under $5 \%$ oxygen.

Estimation of developmental competence of early embryos by means of noninvasive methods is essential for practical and clinical applications such as humanassisted reproduction, and endangered and domestic animal reproduction. Both the speed of embryo development and the nuclear number may be good indicators of developmental viability of fetuses $[5,6,10,11,13]$. Our data on embryo development and the nuclear number showed conflicting results: faster blastocoel formation with low nuclear numbers in Slc embryos cultured under $5 \%$ oxygen without amino acids. Transfer of embryos into surrogate females must be conducted to clarify developmental competence and oxygen-amino acid interaction.

The culture conditions we employed supported very high levels of in vitro development of inbred C57BL/6J mouse embryos to the blastocyst ( $>85 \%$ ) and expanded blastocyst $(>80 \%)$ stages. Because our results indicate substrain differences in response to culture environments, proper selection of culture conditions is important in the manipulation of embryos in vitro and care must be taken when this inbred strain is used as material for the studies of embryo development and other studies.

\section{References}

1. Bavister, B.D. 1995. Hum. Reprod. Update 1: 91-148.

2. Bavister, B.D. 1988. Theriogenology 29: 143-154.
3. Biggers, J.D., McGinnis, L.K., and Raffin, M. 2000. Biol. Reprod. 63: 281-293.

4. Devreker, F., Hardy, K., Van den Bergh, M., Vannin, A.S., Emiliani, S., and Englert, Y. 2001. Hum. Reprod. 16: 749756.

5. Gardner, D.K. 1998. Theriogenology 49: 83-102.

6. Gonzales, D.S., Pinheiro, J.C., and Bavister, B.D. 1995. J. Reprod. Fertil. 105: 1-8.

7. Ho, Y., Wigglesworth, K., Eppig, J.J., and Schultz, R.M. 1995. Mol. Reprod. Dev. 41: 232-238.

8. Hogan, B., Beddington, R., Costantini, F., and Lacy, E., 1994. pp. 115-126. In: Manipulating the Mouse Embryo, 2nd Ed., Cold Spring Harbor, NY.

9. Johnson M.H. and Nasr-Esfahani M.H., 1994. BioEssay 16: 31-38.

10. Lane, M. and Gardner, D.K. 1994. J. Reprod. Fertil. 102: 305-312.

11. Lane, M. and Gardner, D.K. 1997. J. Assist. Reprod. Genet. 14: 398-403.

12. Lawitts, J.A. and Bigger, J.D. 1993. Methods Emzymol. 225: 153-164.

13. McKiernan, S.H. and Bavister, B.D. 1994. Hum. Reprod. 9: 2123-2129.

14. McKiernan, S.H., Bavister, B.D., and Tasca, R.J. 1991. Hum. Reprod. 6: 64-75.

15. McLaren, A. and Bowman, P. 1973. J. Embryol. Exp. Morphol. 30: 491-498.

16. Miyoshi, I., Ishikawa, K., Kasai, M., and Kasai, N. 1992. Lab. Anim. Sci. 42: 323-325.

17. Mobraaten, L.E. 1986. J. In Vitro Fertil. Embryo Transf. 3: 28-32.

18. Pabon, J.E., Findley, W.E., and Gibbons, W.E. 1989. Fertil. Steril. 51: 896-900.

19. Roudebush, W.E. and Duralia, D.R. 1996. Lab. Anim. Sci. 46: 239-240.

20. Suzuki, H., Kamada, N., Ueda, O., Jishage, K., Kurihara, H., Kurihara, Y., Kodama, T., Yazaki, Y., Azuma, S., and Toyoda, Y. 1994. J. Reprod. Dev. 40: 361-365.

21. Toyoda, Y., Yokoyama, M., and Hosi, T. 1971. Jpn. J. Anim. Reprod. 16: 147-151.

22. Warikoo, P.K. and Bavister, B.D. 1989. Fertil. Steril. 51: 886-889.

23. Whitten, W.K. and Dagg, C.P. 1962. J. Exp. Zool. 148: 173-183.

24. Wood, S.A., Pascoe, W.S., Schmidt, C., Kemler, R., Evans, M.J., and Allen, N.D., 1993. Proc. Natl. Acad. Sci. USA 90: 4582-4585.

25. Zar, J.H. 1996. In: Biostatistical Analysis, 3rd Ed., PrenticeHall, Englewood Cliffs, NJ. 